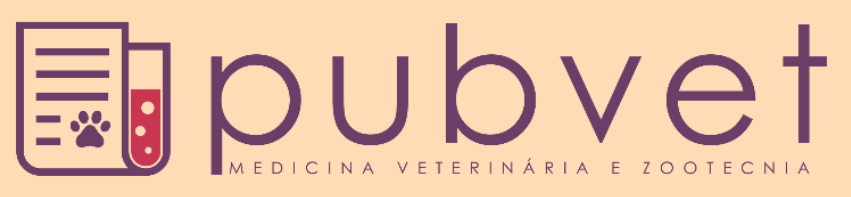

https://doi.org/10.31533/pubvet.v13n9a408.1-3

\title{
Demodiciose canina associada a malasseziose
}

\author{
Daiane Linhares Gonzatto ${ }^{1^{*}}$, Eduarda do Amaral Souza Silva ${ }^{1 \bullet}$, Elber Rodrigues Rezende ${ }^{1}$, \\ Ingryd Muniz de Oliveira ${ }^{1}{ }^{\circ}$, Gabriel Utida Eguchi ${ }^{2}{ }^{\circ}$, Joyce Katiuccia Medeiros Ramos Carvalho ${ }^{3}{ }^{\circ}$ \\ ${ }^{I}$ Acadêmicos de Medicina Veterinária, Universidade Católica Dom Bosco (UCDB) \\ ${ }^{2}$ Médico veterinário do Hospital Veterinário da Universidade Católica Dom Bosco \\ ${ }^{3}$ Mestre, Docente do Curso de Medicina Veterinária da Universidade Católica Dom Bosco (UCDB) \\ *Autor para correspondência, E-mail: daiane_gonzatto@hotmail.com
}

Resumo. A demodiciose, conhecida popularmente como "Sarna Negra" é uma doença de pele, ocasionada pela multiplicação excessiva do ácaro do gênero Demodex. Este trabalho visa relatar o caso de um cão da raça Bulldog Francês, fêmea, de três anos de idade, atendida no Hospital Veterinário da Universidade Católica Dom Bosco, que apresentava lesões na cabeça e nos membros, alopecia localizada, hiperpigmentação, eritema, prurido moderado. Associada a doença, o animal manifestava dermatopatia fúngica, causada pelo fungo Malassezia pachydermatis, no qual, demonstraram sinais clínicos de alopecia regional, cheiro de "ranço", liquenificação crônica nas orelhas, hiperqueratose, otite externa e hipotricose localizada. No exame parasitológico de raspado de pele profundo foram observadas formas de Demodex $s p$. E nesse caso, foi prescrito para o tratamento Simparic, Itraconazol e banhos com shampoo a base de Clorexidine $2 \%$, a fim de evitar recidivas e controlar a doença. Outro procedimento adotado para controle da doença é a retirada da fêmea da reprodução, pois, a mesma não tem cura, apenas melhora clínica.

Palavras chaves: ácaro, canina, demodicose, fungo

\section{Canine demodicosis associated with malasseziosis}

\begin{abstract}
Demodicide, commonly known as "Black Scabies" is a skin disease caused by excessive multiplication of the genus Demodex. This work aims to report the case of a three-year-old Bulldog French dog, attended at the Veterinary Hospital of the Catholic University of Dom Bosco, with lesions on the head and limbs, localized alopecia, hyperpigmentation, erythema, moderate pruritus. Associated with the disease, the animal showed fungal dermatopathy, caused by the fungus Malassezia pachydermatis, in which it showed clinical signs of regional alopecia, rancid smell, and chronic lichenification in the ears, hyperkeratosis, external otitis and localized hypotrichosis. In the parasitological exam of deep skin scrapings forms were observed of Demodex sp. In that case, it was prescribed for the Simparic treatment, Itraconazole and baths with shampoo based on Clorexidine $2 \%$, in order to prevent relapses and control the disease. Another procedure adopted to control the disease is the removal of the females from the reproduction, because it has no cure, only clinical improvement.
\end{abstract}

Keywords: mite, dog, demodicosis, fungus

\section{Demodicosis canina asociada a malasseziosis}

Resumen. La demodiciosis, conocida popularmente como "sarna negra" es una enfermedad de la piel causada por la multiplicación excesiva del ácaro Demodex. Este artículo tiene como objetivo informar el caso de una hembra canina de la raza Bulldog 
Francés de tres años atendido en el Hospital Veterinario de la Universidad Católica Dom Bosco, con lesiones en la cabeza y las extremidades, alopecia localizada, hiperpigmentación, eritema, prurito moderado. Asociado con la enfermedad, el animal manifestó dermatopatía fúngica, causada por el hongo Malassezia pachydermatis, que mostró signos clínicos de alopecia regional, olor rancio, liquenificación crónica del oído, hiperqueratosis, otitis externa e hipotricosis localizada. El examen parasitológico de los restos de piel profunda mostró formas de Demodex sp. En este caso, se prescribieron baños de champú Simparic, itraconazol y clorhexidina al $2 \%$ para prevenir recaídas y controlar la enfermedad. Otro procedimiento adoptado para controlar la enfermedad fue la eliminación de la hembra como reproductora, porque no tiene cura, solo mejora clínica.

Palabras clave: ácaros, caninos, demodex, hongos

\section{Introdução}

A demodiciose, conhecida popularmente como "Sarna Negra" é uma doença de pele, ocasionada pela multiplicação excessiva do ácaro do gênero Demodex; sendo a $D$. canis a espécie mais frequente (Cardoso et al., 2011; Larsson, 1996). Essa doença é causada pela destruição do folículo piloso e, consequentemente, ocorre o entupimento do orifício do folículo (Griffin, 1996; Guillot \& Bond, 1999). É uma dermatopatia inflamatória que está associada a uma deficiência do sistema imunológico. A malasseziose é uma dermatopatia fúngica, causada pelo fungo leveduriforme Malassezia pachydermatis, presente na microbiota normal do animal; porém, quando ocorre desequilíbrio na microbiota do animal causa a proliferação intensa (Girão et al., 2006; Guillot \& Bond, 1999).

\section{Relato de caso}

Foi atendida no Hospital Veterinário da Universidade Católica Dom Bosco (HOVET), uma canina da raça Bulldog Francês, de três anos de idade, pesando $10 \mathrm{~kg}$. Apresentava lesões na cabeça e nos membros, alopecia localizada, hiperpigmentação, eritema, prurido moderado, cheiro de "ranço", liquenificação crônica nas orelhas (Figura 1), hiperqueratose, otite externa e hipotricose localizada (Figura 2). Baseado, na anamnese, sinais clínicos e resultado do exame parasitológico, foi estabelecido o tratamento com Simparic $20 \mathrm{mg} / 10 \mathrm{~kg}$ de peso, por via oral, a cada 30 dias; Itraconazol $10 \mathrm{mg} / \mathrm{kg}$ por via oral, junto com a alimentação, a cada 24 horas, durante 15 dias e banhos com shampoo a base de Clorexidine 2\%, a cada 7 dias. Indicado retorno para reavaliação clínica em 30 dias.

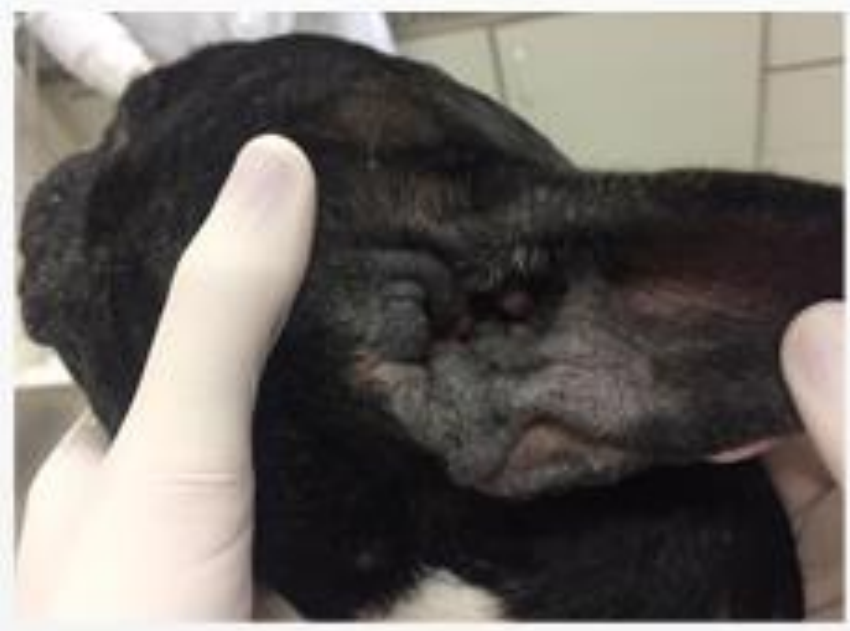

Figura 1. Cão da raça Bulldog Francês, fêmea, apresentando liquenificação em região de ouvido.

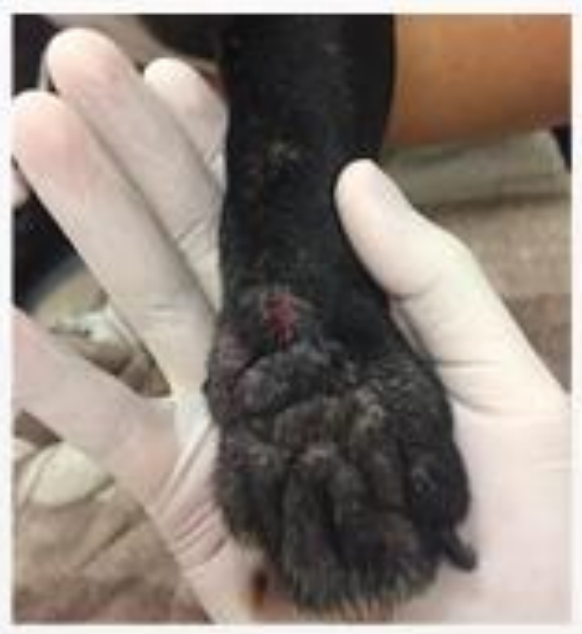

Figura 2. cão da raça Bulldog Francês, fêmea, apresentando hipotricose localizada na porção distal em membros.

\section{Resultado e discussão}

Após o retorno, devido à característica da sarna em permanecer no folículo piloso foi realizado o exame de raspado profundo de pele. E observado pela microscopia ótica a presença da forma adulta de 
Demodex canis. Essa enfermidade pode atingir todas as raças de cães e de todas as idades, apesar de que, os filhotes na maioria das vezes são os mais acometidos (Nóbrega, 2018; Santos et al., 2009). Durante o exame clínico foi identificada a presença de Malassezia pachydermatis, e nesse caso, foram aconselhados os exames de citologia e cultura fúngica (meio de Dickson), exames importantes para se chegar ao diagnóstico.

Como procedimento foi indicado para controle da sarna Demodex canis a retirada da fêmea da reprodução por se tratar de uma doença que não tem cura, apenas melhora sintomatológica. Essa enfermidade está associada à deficiência do sistema imunológico do animal, em decorrência de alguns fatores tais como: estresse, mudança de ambiente, alimentação inadequada, verminoses. Com o animal imunossuprimido, houve também o crescimento exagerado do fungo Malassezia pachydermatis. $\mathrm{O}$ tratamento estabelecido tem a finalidade de evitar recidivas e controlar a doença.

\section{Referências bibliográficas}

Cardoso, M. J. L., Machado, L. H. A., Melussi, M., Zamarian, T. P., Carnielli, C. M. \& Júnior Ferreira, J. C. M. (2011). Dermatopatias em cães: revisão de 257 casos. Archives of Veterinary Science, 16(2):66-74.

Girão, M. D., Prado, M. R., Brilhante, R. S. N., Cordeiro, R. A., Monteiro, A. J., Sidrim, J. J. C. \& Rocha, M. F. G. (2006). Malassezia pachydermatis isolated from normal and diseased external ear canals in dogs: a comparative analysis. The Veterinary Journal, 172(3):544-548.

Griffin, C. E. (1996). Malassezia paronychia in atopic dogs. Paper presented at the Proceedings of The 12th Annual AAVD and ACVD Members' Meeting. Las Vegas.

Guillot, J. \& Bond, R. (1999). Malassezia pachydermatis: a review. Medical Mycology, 37(5):295-306.

Larsson, C. E. (1996). Dermatopatias alérgicas-hipersensibilidade medicamentosa (HM). Clínica Veterinária, 5(1):351-355.

Nóbrega, B. G. (2018). Estudo retrospectivo de demodicose e escabiose em cães atendidos no Hospital Veterinário de Areia - PB, Campus II Areia, Paraíba: Universidade Federal da Paraíba.

Santos, L. M., Machado, J. d. A. C. \& Neves, M. (2009). Demodicose canina: revisão de literatura. Revista Cientifica Eletrônica de Medicina Veterinária, 7(12):1-5.

Recebido: 20 de junho, 2019.

Aprovado: 17 de agosto, 2019.

Publicado: 31 de outubro, 2019.

Licenciamento: Este artigo é publicado na modalidade Acesso Aberto sob a licença Creative Commons Atribuição 4.0 (CC-BY 4.0), a qual permite uso irrestrito, distribuição, reprodução em qualquer meio, desde que o autor e a fonte sejam devidamente creditados. 